\title{
REAL-SPACE PAIR MODEL PREDICTIONS FOR THE NMR BEHAVIOUR IN HIGH- $T_{\mathrm{c}}$ CUPRATES
}

\author{
S.I. MUKHIN* \\ Kamerling Onnes Laboratory, Leiden University \\ P.O. Box 9506, 2300 RA Leiden, Netherlands
}

\begin{abstract}
A model for real-space singlet pairs, hopping on the quasi 2D antiferromagnetic background of localized spins is further analysed. It is shown that lattice vibrations (if any), which break the symmetry of the in-plane oxygen position with respect to the nearest neighboring copper sites, may strongly influence both the magnitude and the temperature dependence of the ${ }^{17} \mathrm{O}$ NMR relaxation rate. For the $\mathrm{Y}$ site a situation is analogous, although the different modes may contribute to the effect. A relation of the results obtained to the Millis-Monien-Pines analysis is discussed. The validity of the mean-field random phase approximation for the description of the $2 \mathrm{D}$ antiferromagnet used previously is analysed. It is shown that the 2D fluctuations of the phase of the (bond) mean field parameter lead to the power-law decay with the distance of the corresponding correlation function (which remains finite in the mean field approximation). The spectrum of the phase fluctuations is found to be propagative in the one-loop approximation. An interaction of the real-space singlet pairs with the long-wavelength phase fluctuations is discussed.
\end{abstract}

PACS numbers: $75.10 . \mathrm{Jm}$

\section{The model and the formalism}

It is by now generally accepted that strong Coulomb correlations should play an essential role in the physics of the layered copper oxides [4]. On the basis of the purely insulating and antiferromagnetic behavior of the undoped compounds, one expects an (almost) localized nature of the $\mathrm{Cu}^{2+}$ spins also at low doping concentrations (see e.g. [5]). To get some insight in the possible mechanisms underlying the unconventional NMR behaviour of the doped cuprates [6], a model of real-space pairs (RSP's) interacting with the local magnetic order has been proposed [1]. It is implied that the RSP's are formed far above $T_{\mathrm{c}}$ and are responsible for both the normal and superconducting charge transport in the high-temperature superconductor (HTS) cuprates (see [7]).

It is assumed that only the singlet states (singlets) formed by a localized $\mathrm{Cu}^{2+}$ spin and a doped hole spin are allowed. Then, an RSP is supposed to consist

* On leave of absence from Moscow Institute of Steel and Alloys Theoretical Physics Dept., Leninskii prospect 4, 117936 Moskva, Russia. 
of two such singlets occupying adjacent plaquettes of the square $\mathrm{Cu}^{2+}$ lattice in the $\mathrm{CuO}_{2}$ plane. The presence of the RSP causes a local softening of the magnetic exchange interactions between the localized spins, because each of the two holes forming an RSP has its spin bound in the singlet state with the localized $\mathrm{Cu}^{2+}$ spin on the corresponding plaquette. Therefore, these two spins are no longer available for the exchange interactions with their neighbours in the presence of an RSP.

The model Hamiltonian for the RSP-localized spins system has the form [1]:

$$
\begin{aligned}
& H=J \sum_{\langle i, j\rangle} S_{i} \cdot S_{j}\left(1-\hat{\rho}_{i j}\right)+H_{h}, \\
& H_{h}=-\mu \sum_{\langle i, j\rangle} \hat{\rho}_{i j}-t_{\perp} \sum_{\langle i j\rangle,\left\langle i^{\prime} j^{\prime}\right\rangle}\left(b^{+}{ }_{i j} b_{i^{\prime} j^{\prime}}+\text { h.c. }\right)+U \sum_{\langle i j\rangle} \hat{\rho}_{i j}^{2}+H_{z},
\end{aligned}
$$

where $\hat{\rho}_{i j}=b^{+}{ }_{i j} b_{i j} ;\left[b_{i j}, b^{+}{ }_{i^{\prime} j^{\prime}}\right]=\delta_{i j, i^{\prime} j^{\prime}}$; and $S_{i}$ is the spin-1/2 operator of the localized $\mathrm{Cu}^{2+}$ spin on site $i$ (we avoid vector notation for the site indexes in all the formulae for the sake of simplicity). It is supposed that the summation in Eq. (1) goes over an infinite sequence of $2 D$ layers which are not mutually coupled magnetically. It seems to be legitimate to exploit here the strong spatial anisotropy of the cuprates (at least the La-based compounds), which results in the estimate $J^{\prime} / J \approx 10^{-5}$ (see e.g. [5]).

The operators $b_{i j}^{+}, b_{i j}$ in Eq. (2) are the creation and annihilation operators of the RSP, which occupies two adjacent plaquettes of the $\mathrm{CuO}_{2}$ lattice centered at $i$ and $j$, and are subjected to the constraint $\hat{\rho}_{i j}=0,1$ (i.e. $U \rightarrow \infty$ is implied in the Hamiltonian in Eq. (2)); $\mu$ is the chemical potential of the RSP's, and $t_{\perp}$ and $H_{z}$ are the RSP intralayer hopping integral and the interlayer hopping contribution to the RSP's IIamiltonian respectively.

Taking into account the onsite constraint for $\hat{\rho}_{i j}$, we rewrite the interaction part of the Hamiltonian Eq. (1) in the completely biquadratic form (see [1]):

$$
\begin{aligned}
& H_{\mathrm{int}}=-\frac{1}{2} J \sum_{i j} A_{i j}^{+} A_{i j}\left(1-\hat{\rho}_{i j}\right)^{2} \\
& A_{i j}^{+}=\sum_{\sigma=1,2} a_{i \sigma}^{+} a^{+}{ }_{j \sigma}, \quad A_{i j}=\sum_{\sigma=1,2} a_{i \sigma} a_{j \sigma},
\end{aligned}
$$

where the Schwinger boson representation for the localized spins-1/2 [3] is used. The Schwinger boson operators $a_{i \sigma}\left(a_{i \sigma}^{+}\right)(\sigma=1,2)$ obey the Bose commutation relations and are subjected to the local constraint $\sum_{\sigma=1,2} a_{i \sigma}^{+} a_{i \sigma}=1$.

Then, after the Stratonovich-Hubbard decoupling of the interaction term in Eq. (3) the effective action takes the form [1]:

$$
\begin{aligned}
S= & \int_{0}^{\beta} \mathrm{d} \tau\left[\frac{1}{2} \sum_{i, \sigma}\left(\bar{a}_{i \sigma} \dot{a}_{i \sigma}-\dot{\bar{a}}_{i \sigma} a_{i \sigma}\right)+\frac{2}{\tilde{J}} \sum_{\langle i j\rangle} \bar{Q}_{i j} Q_{i j}\right. \\
& \left.+\sum_{\langle i j\rangle}\left(\bar{Q}_{i j} A_{i j}+Q_{i j} \bar{A}_{i j}\right)\left(1-\delta \hat{\rho}_{i j}\right)+\sum_{i \sigma} \lambda_{i}\left(\bar{a}_{i \sigma} a_{i \sigma}-\frac{1}{2}\right)\right],
\end{aligned}
$$

where $\delta \hat{\rho}_{i j}=\left(\hat{\rho}_{i j}-n\right)(1-n)^{-1} ; \tilde{J}=J(1-n)^{2}$, and $n$ is the RSP density. Next, we integrated out the spin degrees of freedom to the one-loop accuracy in the 
MF approximation for the Stratonovich fields, i.e. taking $Q_{i j}=Q$ and $\lambda_{i}=\lambda$. The resulting inter-RSP interaction (it proved to be attraction) was obtained as a result of such a procedure. Using this result, the interaction contribution to the Gibbs free energy $\Xi$ of the system in the random phase approximation was found to be [1]:

$$
\Xi_{\mathrm{int}}^{\mathrm{RPA}}=-\frac{T Q^{2}}{4} \int_{0}^{1} \sum_{\boldsymbol{q}, \omega} D(\boldsymbol{q}, \omega) \Pi(\boldsymbol{q}, \omega)\left[1-\frac{x Q^{2}}{4} D(\boldsymbol{q}, \omega) \Pi(\boldsymbol{q}, \omega)\right]^{-1} \mathrm{~d} x
$$

where $\Pi(q, \omega)$ is the Fourier component of the RSP's density-density correlation function, while $D(\boldsymbol{q}, \omega)$ is the mean-field correlation function of the fluctuations of the field $A_{i j}^{+}+A_{i j}$. Finally, $\omega$ is the Matsubara frequency and $Q$ is the mean-field parameter to be determined self-consistently (together with the antiferromagnetic correlation length $\xi(T)$ ) by minimizing the Gibbs free energy.

Here we remind some results obtained in [1]. In the interval of parameter $\left(n t_{\perp}, T\right) \ll \tilde{J}$, the self-consistent equations obtained by minimizing the Gibbs free energy $\Xi$ take the form

$$
\begin{aligned}
& n=\frac{1}{2 N} \sum_{k}^{\prime} N\left(\varepsilon_{k}\right)-\frac{3\left(1-\eta^{2}\right)}{\pi} \ln \left(1-\frac{n(\mu)}{\pi\left(1-\eta^{2}\right)}\right), \\
& 1=\frac{\tilde{J}}{N} \sum_{k} \operatorname{coth}\left(\frac{\beta \omega_{k}}{2}\right) \frac{1}{\omega_{k}}-\frac{3 T \eta^{2}}{\pi \tilde{J}} \ln \left(1-\frac{n(\mu)}{\pi\left(1-\eta^{2}\right)}\right),
\end{aligned}
$$

where $N\left(\varepsilon_{k}\right)=\left[\exp \beta\left(\varepsilon_{k}-\mu\right)-1\right]^{-1} ; \varepsilon_{k}=k^{2} / 2 m_{\perp}$ and $m_{\perp}$ is the RSP mass for inplane motion $\left(t_{\perp}^{-1} \sim m_{\perp} a^{2}\right) ; \omega_{k} \approx 2 \tilde{J}\left(1-\eta^{2} \gamma_{k}^{2}\right)^{\frac{1}{2}}$ is the magnetic excitation energy; $\eta^{2} \sim 1-a^{2} / \xi^{2} ; \gamma_{k}=\frac{1}{4} \sum_{a} \exp (\mathrm{i} k \cdot a)$ ( $a$ spans over the nearest neighbouring sites of the square lattice); and $n(\mu)=\pi T /\left(2 t_{\perp}\right) \exp (\mu / T)$. Quasi-twodimensionality of the RSP system is assumed: $t_{z} \ll t_{\perp}$, where $t_{z}$ is the interplanar RSP hopping integral. The symbol $\sum$ ' means summation over the first RSP's Brillouin zone, and $N$ is the number of the square lattice sites. The logarithmic terms in Eqs. (7), (8) are due to $\Xi_{\text {int }}^{\text {RPA }}$. Then we have

$$
\begin{array}{ll}
\xi / a \sim\left(1-\eta^{2}\right)^{-1 / 2} \sim \exp (\nu \tilde{J} /(2 T)), & T^{*} \ll T \ll \tilde{J}, \\
\xi / a \sim(\tilde{J} /(n T))^{1 / 2}, \quad n(T) \sim n T / \tilde{J}, & T^{* *} \ll T \ll T^{*},
\end{array}
$$

where $T^{*} \sim \nu \tilde{J} / \ln (1 / n)$ and $\nu \approx 2.2$ in the RPA [8]. Here $n(T)$ means $n(\mu(T))$, and the second relation in Eq. (9) shows that below $T^{*}$ the RPA solution exists if only the density of "free bosons" (RSP's) scales with the $(a / \xi(T))^{2}$. This may indicate that to keep the system in the homogeneous state at $T<T^{*}$, the effective number of bosons contributing to the long-range RSP density fluctuations should decrease. This in turn would be expected to happen if the RSP's gather in some kind of mobile "droplets", which are responsible then for the long-range density fluctuations (which indeed is seen by the Monte Carlo simulations performed for the present model [9]). Finally, at $T<T^{* *} \sim n \tilde{J}$ the minimum magnetic excitation energy $\omega \sim J a / \xi(T)$ becomes greater than $T$ and system likely fails to approach the limit $\xi \rightarrow \infty$ at $T \rightarrow 0$. 
The physical meaning of the crossover at $T^{*}$ in our model follows from Eq. (1). Namely, an RSP has lower potential energy in the regions with suppressed absolute value of the short-range order parameter $\left\langle\boldsymbol{S}_{i} \cdot \boldsymbol{S}_{j}\right\rangle$. Due to the thermal fluctuations, the size of such a region around the RSP is not greater than $\xi(T)$, and reaches the inter-RSP distance $a / \sqrt{n}$ at $T \approx T^{*}$. Then, the strong inter-particle attraction appears in the "gas" of the RSP's. The logarithmic terms in Eqs. (7), (8) become singular at this temperature. Therefore, the RSP-spin system has to reduce the increase in $\xi(T)$ in a self-consistent manner in order to remain in a homogeneous state also at $T<T^{*}$ ( the phase separation is avoided when at least $\left.-J\left\langle S_{i} \cdot S_{j}\right\rangle_{T^{*}}<t_{\perp}\right)$.

\section{NMR behaviour in the RSP-spin model}

In this section we shall apply the temperature dependencies for $\xi(T)$ found above to obtain the resultant behaviour of the NMR characteristics in the RSP-spin model. Since we are only concerned with the qualitative picture, we use here the simplest form of the hyperfine coupling Hamiltonian

$$
H_{\mathrm{hf}}=A \sum_{i} \boldsymbol{S}_{i} \cdot \boldsymbol{I}_{i}
$$

Here $A$ is the hyperfine "coupling constant" and $\boldsymbol{I}_{i}$ is the nuclear spin operator at the site $i$. In what follows only the onsite interaction is considered between a localized electronic spin and the corresponding nuclear spin. The spin lattice sites in the present model then correspond to the $\mathrm{Cu}^{2+}$ sites. However, to model the NMR behaviour at the inplane oxygen sites, one may use the following Hamiltonian:

$$
H_{\mathrm{hf}}^{\mathrm{O}}=A^{\prime} \sum_{i, \boldsymbol{a}}\left(S_{i+\frac{a}{2}}+S_{i-\frac{a}{2}}\right) \cdot I_{i}
$$

where the summation with respect to the electronic spins is now over the "oxygen sites" in the $\mathrm{CuO}_{2}$ plane (see [10]). Therefore, the results obtained below may easily be extended to the oxygen-NMR by the introduction of the proper form factors in the hyperfine interaction Hamiltonian.

As usual [11], the Knight shift $K$, and the nuclear spin-relaxation rate $1 / T_{1}$, are related to the Fourier components $\chi(q, \omega)$ of the susceptibility of the localized spin system

$$
\begin{aligned}
& K(T) \sim \chi(q=0, \omega=0), \\
& \frac{1}{T_{1} T} \sim A^{2} \int \mathrm{d}^{2} \boldsymbol{q}\left[\frac{\operatorname{Im} \chi(\boldsymbol{q}, \omega)}{\omega}\right]_{\omega \rightarrow 0} .
\end{aligned}
$$

In case an isotropic magnetic exchange integral is assumed, one has [12]:

$$
\chi^{\mathrm{Cu}}(\boldsymbol{q}, \omega)=\left\{-\mathrm{i} \Theta\left(t-t^{\prime}\right)\left\langle\left[S^{z}(r, t), S^{z}\left(\boldsymbol{r}^{\prime}, t^{\prime}\right)\right]\right\rangle\right\} \boldsymbol{q , \omega},
$$




$$
\begin{aligned}
& \chi^{\mathrm{O}}(\boldsymbol{q}, \omega)=\left\{-\mathrm{i} \Theta\left(t-t^{\prime}\right)\left\langle\left[\left(S^{z}(r, t)+\left(1+C^{\prime} u_{r, t}\right) S^{z}(r+a, t)\right),\right.\right.\right. \\
& \left.\left.\left.\left(S^{z}\left(r^{\prime}, t^{\prime}\right)+\left(1+C^{\prime} u_{r^{\prime}, t^{\prime}}\right) S^{z}\left(r^{\prime}+a^{\prime}, t^{\prime}\right)\right)\right]\right\rangle\right\}_{q, \omega}, \\
& \Theta(x)= \begin{cases}1 & \text { if } x>0, \\
0 & \text { otherwise, }\end{cases}
\end{aligned}
$$

where $u_{r, t}(\ll 1)$ mimics the symmetry breaking lattice mode, and $C^{\prime}$ is the coupling constant. Then, the Knight shift $K$ and the nuclear spin-relaxation rate $1 / T_{1}$, are related to the Fourier components $\chi(q, \omega)$ of the susceptibility in the usual way [11]:

$$
\begin{aligned}
& K^{\mathrm{Cu}}(T) \sim \chi^{\mathrm{Cu}}(q=0, \omega=0), \\
& \left(\frac{1}{T_{1} T}\right)^{\mathrm{Cu}} \sim A^{2} \int \mathrm{d}^{2} q\left[\frac{\operatorname{Im} \chi^{\mathrm{Cu}}(q, \omega)}{\omega}\right]_{\omega \rightarrow 0}, \\
& \left(\frac{1}{T_{1} T}\right)^{\mathrm{O}} \sim{A^{\prime}}^{\prime 2} \int \mathrm{d}^{2} q\left[\frac{\operatorname{Im} \chi^{\mathrm{O}}(q, \omega)}{\omega}\right]_{\omega \rightarrow 0} .
\end{aligned}
$$

We see that at $u_{r, t} \equiv 0$ the result for oxygen site differs from that one for the copper site by a well-known form factor $F(q)=1-\frac{1}{2}\left(\cos q_{x} a+\cos q_{y} a\right)$ being inserted into the integrand in Eq. (15) [2] when $\chi(q, \omega)$ taken from Eq. (13).

Then, in the present model, $\chi^{\mathrm{Cu}}(q, \omega)$ takes the form

$$
\begin{aligned}
& \chi^{\mathrm{Cu}}(q, \omega)=\frac{1}{4 N} \sum_{k}\left\{\frac{\cosh ^{2}\left(\Theta_{k}+\Theta_{k+q}\right)\left[\operatorname{coth}\left(\omega_{k+q} / 2 T\right)-\operatorname{coth}\left(\omega_{k} / 2 T\right)\right]}{\omega_{k}-\omega_{k+q}+\omega+i \delta}\right. \\
& \left.+\frac{\sinh ^{2}\left(\Theta_{k}+\Theta_{k+q}\right)\left[\operatorname{coth}\left(\omega_{k+q} / 2 T\right)+\operatorname{coth}\left(\omega_{k} / 2 T\right)\right]\left(\omega_{k}+\omega_{k+q}\right)}{\left(\omega_{k}+\omega_{k+q}\right)^{2}-(\omega+\mathrm{i} \delta)^{2}}\right\},(17)
\end{aligned}
$$

where $q$ is measured from the corner of the Brillouin zone $(\pi / a, \pi / a)$ of the Cu-layer, and $\sinh \left(2 \Theta_{k}\right)=\eta \gamma_{k}\left(1-\eta^{2} \gamma_{k}^{2}\right)^{-\frac{1}{2}}$. Now, it follows from Eq. (14) that $u_{r, t}$-induced contribution to $\chi^{\circ}(q, \omega)$ reads (in the Matsubara representation):

$$
\Delta \chi^{\mathrm{o}}(\boldsymbol{q}, \omega) \sim T \sum_{\Omega} \chi^{\mathrm{Cu}}(\boldsymbol{q}, \Omega) D(\omega-\Omega),
$$

where we take the $u_{r, t}$ Green function $D$ in the local mode form

$$
D(\omega)=\frac{2 \omega_{0}}{\omega_{0}^{2}+\omega^{2}}
$$

and $\omega_{0}$ is the local mode frequency. Using now Eqs. (15)-(19) one finds

$$
\begin{aligned}
& K^{\mathrm{Cu}} \sim \frac{T}{4 \pi \tilde{J}^{2}} \ln \frac{T \xi}{\tilde{J} a}, \quad\left(\frac{1}{T_{1} T}\right)^{\mathrm{Cu}} \sim \frac{A^{2} T \xi}{\tilde{J}^{3} a}, \\
& \left(\frac{1}{T_{1} T}\right)_{0}^{\mathrm{O}} \sim \frac{A^{\prime 2} C^{2} T^{2}}{\tilde{J}^{4}},
\end{aligned}
$$




$$
\Delta\left(\frac{1}{T_{1} T}\right)^{\circ} \sim \frac{A^{\prime 2} C^{\prime 2} T}{\tilde{J}^{3}} \times\left\{\begin{array}{cl}
\frac{\tilde{J}}{\omega_{0}} \frac{T}{\omega_{0}}, & \tilde{J} a / \xi \ll \omega_{0} \ll T, \\
\frac{T}{\omega_{0}} \frac{\xi}{a}, & \omega_{0} \ll \tilde{J} a / \xi \ll T .
\end{array}\right.
$$

Direct comparison of the results of Eqs. (21) and (22) shows that a coupling to the local soft vibration mode via deformation dependent oxygen-copper exchange integral, may change drastically both the magnitude and the temperature dependence of the ${ }^{17} \mathrm{O}$ NMR relaxation rate with respect to the "rigid lattice" approximation discussed by Millis, Monien, Pines (MMP) [2]. An analogous conclusion applies to the $\mathrm{Y}$ site NMR, where the symmetry of the corresponding form factor $F(q)=\left(1-\cos q_{x} a\right)\left(1-\cos q_{y} a\right)$ (see e.g. [2]) may be broken in a similar fashion to the inplane oxygen case (though the coupled vibration modes might be different). Therefore, this effect may play an important role in the distinctive temperature behaviour of the ${ }^{17} \mathrm{O}$ and ${ }^{89} \mathrm{Y}$ NMR characteristics, which was observed recently in the experimental study of the YBCO compound [13].

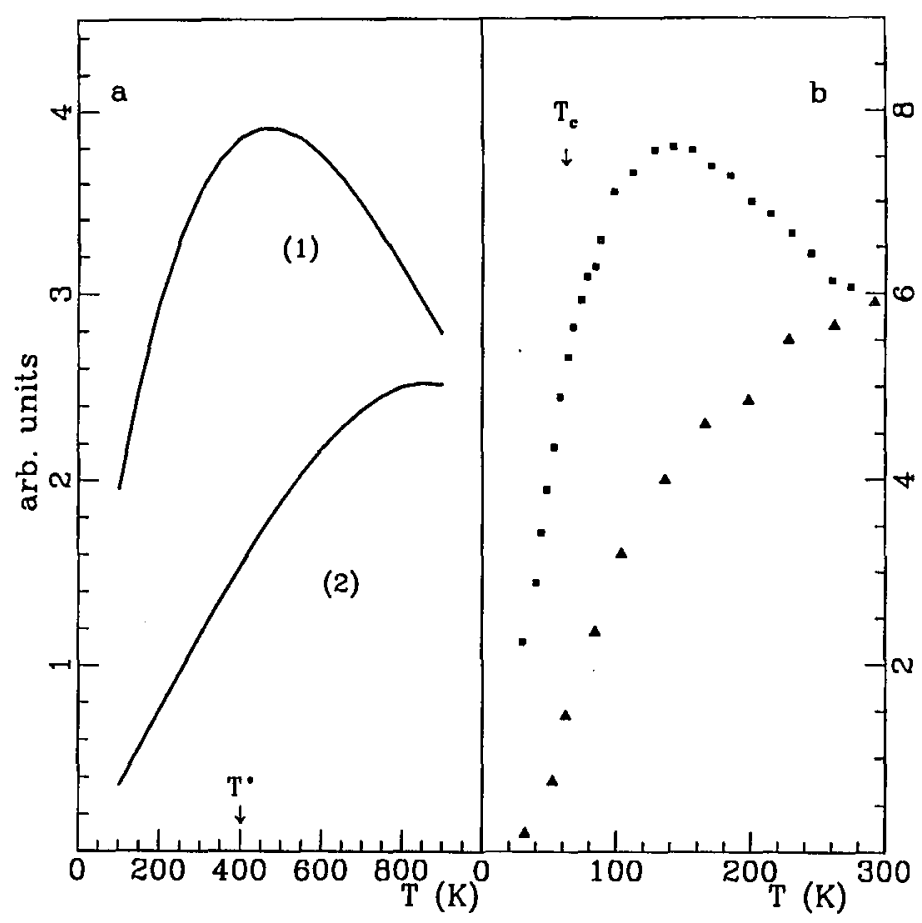

Fig. 1. (a) Schematic plots of the temperature dependencies of $1 / T_{1} T$ (line 1), and $K(T)$ (line 2) predicted for the sector: $n t_{\perp} \ll \tilde{J} ; n=0.01, J=1000 \mathrm{~K}$. Note that for this set of parameters $T^{*} \approx 400 \mathrm{~K}, T^{* *} \approx 10 \mathrm{~K}$; (b) $1 / T_{1} T$ (solid squares), and $K(T)$ (solid triangles) temperature dependencies measured by (Takigawa et al. 1991) in the $\mathrm{YBa}_{2} \mathrm{Cu}_{3} \mathrm{O}_{6.63}$ compound $\left(T_{\mathrm{c}}=62 \mathrm{~K}\right)$. 
The schematic plots for $K^{\mathrm{Cu}}(T)$ and $1 / T_{1}^{\mathrm{Cu}} T$ obtained by means of Eqs. (20) and results for the $\xi(T)$ presented in Eq. (8) (see [1]), together with the experimental data (by Takigawa et al. 1991) in $\mathrm{YBa}_{2} \mathrm{Cu}_{3} \mathrm{O}_{6.63}$ are shown in Fig. 1. We note here that the value $T^{*} \approx 400 \mathrm{~K}$ used in the plots of Fig. 1a is obtained from the estimate after Eq. (8) using the parameters $n=0.01$ and $\tilde{J}=1000 \mathrm{~K}\left(\tilde{J} \gg n t_{\perp}\right)$. Comparing with the experimental results in Fig. $1 \mathrm{~b}$ on $\mathrm{YBa}_{2} \mathrm{Cu}_{3} \mathrm{O}_{6.63}$, for which it is believed that $n \approx 0.05$ (hole density $\approx 0.10$ ) and $J=1500 \div 2000 \mathrm{~K}$, one notices a factor of four difference in the quantitative predictions from our model (the qualitative agreement is obvious from Fig. 1). Within the RPA improved estimates of $T^{*}$ could only change its value by about $20 \div 30 \%$. However, we note that the RPA does not take into account the suppression of the local (on-site) magnetic moment due to the enhancement of the fluctuations by the doping with the mobile holes. Therefore, one expects a considerable reduction of the value of $\nu$ in the aforementioned estimate, in the doped samples with respect to the value $\nu \approx 2.2$ taken from the undoped case [8].

\section{Fluctuations correction to the MF picture}

The spin-spin correlation function $\left\langle\boldsymbol{S}_{\boldsymbol{O}} \cdot \boldsymbol{S}_{\boldsymbol{r}}\right\rangle$ decays exponentially with $\boldsymbol{r}$ at finite temperatures in the MF approximation discussed so far [3]: $\left\langle\boldsymbol{S}_{\boldsymbol{O}} \cdot \boldsymbol{S}_{\boldsymbol{r}}\right\rangle \sim$ $(\xi / r) \exp (-r / \xi)$. The long-range spin ordering takes place in MF only at $T=0$ $(\xi(T=0)=\infty)$. These facts are in correspondence with the $2 \mathrm{D}$ nature of the antiferromagnetic system under consideration. Nevertheless, there is a "hidden" long-range order imposed by the MF approximation. Namely, the MF relation $Q_{i j} \equiv Q$ causes $\left\langle A_{i j} A_{i^{\prime} j^{\prime}}\right\rangle \rightarrow Q^{2}$ at $\left|r_{i j}-r_{i^{\prime} j^{\prime}}\right| \rightarrow \infty$. The situation is changed by allowing for the phase fluctuations, i.e. when we take $Q_{i j} \rightarrow Q \exp \left[i \phi_{i j}\right]$. Then, to the second order in $\phi_{i j}$ one has the following extra term in the spin Hamiltonian (here we neglect the RSP induced corrections yet):

$$
H_{\phi}=Q \sum_{(i, j)}\left[\left(-\mathrm{i} \phi_{i j}-\frac{\phi_{i j}^{2}}{2}\right) A_{i j}+\left(\mathrm{i} \phi_{i j}-\frac{\phi_{i j}^{2}}{2}\right) A_{i j}^{+}\right] .
$$

Now we integrate out the $a_{i \sigma}, a_{i \sigma}$ fields (in the one-loop approximation) to get the effective action $S_{\phi}$ for the phase $\phi$, from which the propagator of the $\phi$-fluctuations $D_{\phi}$ can be found

$$
\begin{aligned}
& S_{\phi}=\sum_{\omega, \boldsymbol{q}} \phi_{\omega, \boldsymbol{q}} \phi_{-\omega,-\boldsymbol{q}} D_{\phi}^{-1}(\boldsymbol{q}, \omega), \\
& D_{\phi}^{R}(\boldsymbol{q}, \Omega)=\frac{16 \pi \tilde{J}^{2}\left(1-\eta^{2}\right)}{T} \frac{1}{\left(q s_{\phi}\right)^{2}-(\Omega+\mathrm{i} \delta)^{2}}, \quad 0<q<\xi^{-1} \\
& s_{\phi} \sim \tilde{J} a \sqrt{1-\eta^{2}}, \quad \delta \rightarrow 0^{+} .
\end{aligned}
$$

From Eq. (24) it follows that, in addition to the MF spin-wave like spectrum with the gap $\Delta=\omega_{q \rightarrow 0} \sim \tilde{J} a / \xi$ found in [3], there are also the lower energy (phase) excitations inside the MF gap. These last ones possess a sound-like spectrum with the effective velocity $s_{\phi} \sim s a / \xi$, where $s \sim \tilde{J} a$ is the usual (mean-field) spin 
velocity. The physical reason for such an effective suppression of the velocity $s_{\phi}$ with respect to $s$ might be explained as follows. Namely, the phase fluctuations found here play the role of the hydrodynamic modes of the 2D quantum Heisenberg antiferromagnet (QHAF). Therefore, the effective media seen by these excitations consist of the correlated clusters of the size $\sim \xi$ ("block-spins") interacting with each other with the characteristic energy ("block exchange integral") of the order of the gap $\Delta$ in the spin-wave MF spectrum, which in turn serves as the effective "rigidity" for the hydrodynamic phase fluctuations. Then, a substitution of $\tilde{J}$ by $\omega_{q \rightarrow 0}$ in the expression for the velocity $s$ gives directly $s_{\phi}$ value.

The presence of such hydrodynamic excitations is not surprising by two reasons. First, the effective action for the Stratonovich fields $S_{\mathrm{eff}}(Q, \bar{Q} ; \lambda)$, which might be derived from Eq. (4) by integrating out the fields $a_{i \sigma}, a_{i \sigma}^{+}$, is invariant with respect to the homogeneous phase shift $Q \rightarrow Q \exp (\mathrm{i} \phi)$ [3]. Therefore, the $\phi$-spectrum should be gapless at $q \rightarrow 0$. Second, since there is a gap $\Delta \sim \tilde{J} a / \xi$ in the MF spin excitations spectrum, $\omega_{k} \approx 2 \tilde{J}\left(1-\eta^{2} \gamma_{k}^{2}\right)^{\frac{1}{2}}$, the phase fluctuations of the MF parameter are propagative in the range of the energies inside the gap $\Omega_{q}<\Delta$ (the damping rate of this excitations due to the scattering at the spin-waves is of the order of $\omega^{3 / 2} / \sqrt{s q}$ when $\omega \sim s_{\phi} q \ll s q$, again in the one-loop approximation).

Now, taking into account the phase fluctuations we obtain

$$
\begin{aligned}
& \left\langle A_{i j}(\tau) A_{i^{\prime} j^{\prime}}(\tau)\right\rangle \approx Q^{2} \exp \left(-\frac{1}{2}\left\langle\left(\phi_{i j}(\tau)-\phi_{i^{\prime} j^{\prime}}(\tau)\right)^{2}\right\rangle\right) \\
& \approx Q^{2} \exp \left(-\frac{T}{4 N} \sum_{\Omega,|k|<k_{0}} D_{\phi}(q, \Omega)\left|\exp \left(\mathrm{i} k \cdot r_{i j}\right)-\exp \left(\mathrm{i} k \cdot r_{i^{\prime} j^{\prime}}\right)\right|^{2}\right),
\end{aligned}
$$

where $D_{\phi}(q, \Omega)$ is taken in the Matsubara representation, and $k_{0} \sim \xi^{-1}$. This leads to the following result (analogous to that one first obtained for the 2D superfluid Bose systems [14]):

$$
\left\langle A_{i j}(\tau) A_{i^{\prime} j^{\prime}}(\tau)\right\rangle \sim Q^{2}\left|r_{i j}-r_{i^{\prime} j^{\prime}}\right|^{-\alpha}, \quad \alpha=\frac{8 \pi \tilde{J}^{2} a^{2}\left(1-\eta^{2}\right)}{s_{\phi}^{2}} \sim 1,
$$

where we have used Eq. (24). It is evident now from Eq. (26) and the expression for $\left\langle S_{\mathbf{0}} \cdot S_{r}\right\rangle$ at the beginning of this section, that both $\left\langle A_{i j} A_{i^{\prime} j^{\prime}}\right\rangle$ and $\left\langle S_{\mathbf{0}} \cdot \boldsymbol{S}_{\boldsymbol{r}}\right\rangle$ vanish when $\left|r_{i j}-r_{i^{\prime} j^{\prime}}\right| \rightarrow \infty$ at finite temperatures. Therefore, we have shown that the phase fluctuations of the Stratonovich (bond) order parameter "restore" the absence of the long-range order in the $2 \mathrm{D}$ system at finite temperature, which was deliberately imposed by MF approximation in $[3,1]$. We hope to address in the forthcoming paper the question of the phase fluctuation corrections to the NMR characteristics calculated above in the MF-RPA.

It is worth noticing here of the apparent infrared divergence caused by the propagative nature of the $D_{\phi}(q, \Omega)$ in the 2D case presently discussed. The situation is reminiscent of the Berezinskii-Kosterlitz-Thouless case [15], so that one would expect to have a kind of low temperature vortex state in the 2D Heisenberg antiferromagnet (QHAF) with the spin 1/2. Though in contradistinction with the widely spread practice of mapping of the low-energy properties of the QHAF on 
the nonlinear $\sigma$ model, see e.g. [16] (which is not rigorously justified for the spin $1 / 2$ ), here we have shown that the proper fluctuating quantity is the phase $\phi_{i, j}$ related to the bond variable $A_{i, j}$ defined in Eq. (3). This problem will be discussed in detail elsewhere in the nearest future.

Now we shall discuss briefly the influence of the RSP's on the picture outlined above. For this purpose one has to substitute $Q_{i j} \rightarrow Q \exp \left[\mathrm{i} \phi_{i j}\right]$ in Eq. (4) and to add the term with $\delta \hat{\rho}_{i j}$ to the $H_{\phi}$ in Eq. (23). Then the dispersion relation for the phase fluctuations found in Eq. (24) is modified in the one-loop approximation with respect to the RSP's term as follows:

$$
\omega_{q}^{2}=s_{\phi}^{2} q^{2}+\Delta_{\mathrm{RSP}}^{2}
$$

where

$$
\begin{aligned}
& \Delta_{\mathrm{RSP}}^{2}=\frac{8 \pi \tilde{J} n(\mu)\left(1-\eta^{2}\right)}{T^{2}}\left[1+\gamma \frac{T^{2}}{\tilde{J}^{2}}\left(1+\gamma_{1}\left(\ln \frac{\xi T}{\tilde{J} a}\right)^{2}\right)\right], \\
& \gamma \sim \gamma_{1} \sim 1
\end{aligned}
$$

and $n(\mu)$ is defined after Eq. (7).

Some important conclusions follow from the above result. Firstly, one notices that the validity of the MF approach used by Arovas and Auerbach [3] for the partition function path integral of the QHAF is better justified for the RSP-doped case, discussed here, below the crossover temperature $T^{*}$. Namely, the second term in the rectangular brackets in Eq. (28) shows the relative strength of the fluctuations of the bond variable $A_{i j}$ with respect to its mean-field value $Q \sim \tilde{J}$. Then, in the temperature interval $\tilde{J} \gg T>T^{*}$, which corresponds to the "as if undoped" (i.e. Arovas-Auerbach like) temperature behaviour of the correlation length $\xi$ (see Eq. (8)), one finds from Eq. (28): $\gamma T^{2} / \tilde{J}^{2}\left(1+\gamma_{1}(\ln \xi T / \tilde{J} a)^{2}\right) \sim \gamma \gamma_{1} \sim 1$. This actually means that the mean-square fluctuation of the bond variable $A_{i j}$ is of the order of its MF value itself $\left\langle\left(\delta A_{i j}\right)^{2}\right\rangle \sim\left\langle A_{i j}\right\rangle^{2} \sim \tilde{J}^{2}$. Thus the MF approach in the undoped case, as well as in the temperature interval mentioned above for the RSP-doped case, is valid only qualitatively. While in the lower temperatures region: $T^{* *}<T<T^{*}$, where $\xi(T)$ depends on $T$ not exponentially due to the presence of the RSP's, one has (see Eq. (8)): $\gamma T^{2} / \tilde{J}^{2}\left(1+\gamma_{1}(\ln \xi T / \tilde{J} a)^{2}\right) \sim$ $\gamma \gamma_{1} T^{2} / \tilde{J}^{2}(\ln T / \tilde{J} n)^{2} \ll 1$. This means that due to suppression of the antiferromagnetic fluctuations below $T^{*}$ in the RSP-doped system the bond variable fluctuations become weaker and MF works well: $\left\langle\left(\delta A_{i j}\right)^{2}\right\rangle \ll\left\langle A_{i j}\right\rangle^{2}$.

Secondly, the presence of the $\Delta_{\text {RSP }}$ in the dispersion relation in Eq. (27) makes the system not infrared divergent any more. Although the last result should be taken cautiously by the following reason. Namely, Eqs. (27), (28) were obtained in the approximation of the small deviations from the homogeneity on the length scale $r \gg \xi$ of the both spin and RSP systems. Meanwhile, a comparison of the characteristic "phase-correlation" length $L_{\phi} \sim s_{\phi} \Delta_{\mathrm{RSP}}^{-1}$ with $\xi$ gives $L_{\phi}<\xi$ at $T<T^{*}$, i.e. the homogeneity condition is not fulfilled. This fact points to the strong coupling of the long-wavelength phase fluctuations to the RSP-density fluctuations in the system below $T^{*}$, which will be discussed in detail elsewhere. 


\section{Conclusions}

Here we have shown that the present RSP-spin model is able to reproduce, at least qualitatively, the NMR behaviour of the layered underdoped high- $T_{\mathrm{c}}$ cuprates, as it is observed experimentally [6]. The physical nature of $T^{*}$ is illuminated (at the MF-RPA level of accuracy and to that extent to which our model is adequate to the reality).

It is demonstrated also that the inplane oxygen NMR relaxation rate may be highly sensitive to the lattice vibrations which break the rigid-lattice symmetry of the $\mathrm{O}$ site in the copper layer. The same is valid for the Y NMR, although the vibrational modes contributing to the effect may be different from the $O$ case.

Further, we have shown that the phase fluctuations of the mean-field (bond) order parameter in the $2 \mathrm{D}$ antiferromagnet restore the absence of the long-range order in the system at finite temperatures. These fluctuations create a spectrum of the propagative states in the gap, which was found in the spectrum of magnetic excitations of the system in the MF approximation [3].

The infrared divergence, which arises (for the 2D system) in the case of the sound-like spectrum of the phase fluctuations given by Eq. (24), indicates the possibility of the Berezinskii-Kosterlitz-Thouless like situation in the 2D Heisenberg antiferromagnet (QHAF) with the spin $1 / 2$, which involves appearance of the vortices of the phase $\phi_{i j}$, discussed in this work.

Finally, the presence of the doped RSP's in the AF system cuts off the infrared divergences in the phase fluctuations and leads to the strong interaction of the RSP-density fluctuations with the phase fluctuations at the large length scale $r>\xi$.

\section{Acknowledgments}

This work is part of the research program of the Stichting voor Fundamenteel Onderzoek der Materie (FOM), which is financially supported by the Nederlandse Organisatie voor Wetenschappelijk Onderzoek (NWO).

The author acknowledges the stimulating support of L.J. de Jongh, as well as informative discussions with D. Reefman.

Some part of this work was accomplished during the visit of the author to Los Alamos in the framework of the Correlated Electron Theory Program at the Center for Materials Science of the Los Alamos National Laboratory.

The author is grateful to D. Pines for pointing out an importance of the understanding of the ${ }^{17} \mathrm{O}$ NMR temperature behaviour in cuprates, and to J.L. Smith for providing with the preprint of his experimental work.

The author would like to thank K.S. Bedell and A.R. Bishop for continuous helpful discussions of the work. The support through the Program and the hospitality of the colleagues of the T-11 group is acknowledged.

\section{References}

[1] S.I. Mukhin, D. Reefman, L.J. de Jongh,; Phys. Scr. 45, 47 (1992); S.I. Mukhin, L.J. de Jongh, Physica C 211, 77 (1993). 
[2] A.J. Millis, H. Monien, D. Pines, Phys. Rev. B 42, 167 (1990).

[3] D.P. Arovas, A. Auerbach, Phys. Rev. B 38, 316 (1988).

[4] P.W. Anderson, Science 235, 1196 (1987); V. Barzykin, L.P. Gor'kov, Phys. Rev. B 46, 3059 (1992).

[5] Y. Endoh, K. Yamada, R.J. Birgeneau, D.R. Gabbe, H.P. Jenssen, M.A. Kastner, C.J. Peters, P.J. Picone, T.R. Thurston, J.M. Tranquada, G. Shirane, Y. Hidaka, M. Oda, Y. Enomoto, M. Suzuki, T. Murakami, Phys. Rev. B 37, 7443 (1988); B. Keimer, R.J. Birgeneau, A. Cassanho, Y. Endoh, R.W. Erwin, M.A. Kastner, G. Shirane, Phys. Rev. Lett. 67, 1930 (1991).

[6] M. Takigawa, A.P. Reyes, P.C. Hammel, J.D. Thompson, R.H. Heffner, Z. Fisk, C. Ott, Phys. Rev. B 43, 247 (1991); C. Berthier, Y. Berthier, P. Butland, W.G. Clark, J.A. Gillet, M. Horvatic, P. Segransan, J.Y. Henry, in: Electronic Properties and Mechanisms of High-T $T_{\mathrm{c}}$ Superconductors, Proc. Int. Workshop, Tsukuba (Japan) 1991, North-Holland, Amsterdam 1992, p. 347.

[7] L.J. de Jongh, Physica C 152, 171 (1988); Solid State Commun. 65, 963 (1988); Physica C 161, 631 (1989); Eur. J. Solid State Inorg. Chem. 27, 221 (1990).

[8] M. Takahashi, Phys. Rev. B 40, 2494 (1989).

[9] D. Reefman, S.I. Mukhin, L.J. de Jongh, Physica $C$ 211, 93 (1993).

[10] B.S. Shastry, Phys. Rev. Lett. 63, 1288 (1989).

[11] T. Moriya, Prog. Theor. Phys. 28, 371 (1962).

[12] A.A. Abrikosov, L.P. Gor'kov, I.E. Dzyaloshinski, Methods of Quantum Field Theory in Statistical Physics, Dover Publ., New York 1975.

[13] M. Takigawa, W.L. Hults, J.L. Smith, Preprint LA-UR-93-880, 1993.

[14] V.N. Popov, Functional Integrals and Collective Excitations, Cambridge University Press, Cambridge 1987.

[15] V.L. Berezinskii, JETP 32, 493 (1971); J.M. Kosterlitz, D.J. Thouless, J. Phys. C 6, 1181 (1973).

[16] S. Chakravarty, B.I. Halperin, D.R. Nelson, Phys. Rev. B 39, 2344 (1989). 